\title{
Adaptive Wireless Vehicular Communication Techniques under Correlated Radio Channels
}

\author{
M. Sepulcre and J. Gozalvez \\ Ubiquitous Wireless Communications Research Laboratory \\ Uwicore, http://www.uwicore.umh.es \\ University Miguel Hernández, Elche, Spain \\ msepulcre@umh.es, j.gozalvez@umh.es
}

\begin{abstract}
Wireless vehicular communication systems require the design and implementation of robust and efficient communication policies to provide the strict quality of service needed by traffic safety applications while guaranteeing the system's scalability. To efficiently guarantee such requirements, the authors have developed an opportunistic-driven adaptive radio resource management mechanism that adapts the transmission parameters based on the operating conditions and traffic safety requirements. Since the performance of adaptive communication techniques can be importantly influenced by channel correlation, this work proposes several compensation policies that allow the proposed opportunistic mechanism to efficiently meet the traffic safety quality of service requirements even under correlated radio channels.
\end{abstract}

Keywords: wireless vehicular communications, adaptive radio resource management, radio channel correlation

\section{INTRODUCTION}

Vehicle-to-Vehicle (V2V) and Vehicle-to-Infrastructure (V2I) communication systems are being developed to enable a wide variety of new applications and services, ranging from cooperative traffic safety applications, to distributed traffic management services or in-vehicle Infotainment services. To achieve these objectives, several important technological challenges yet need to be overcome. In particular, the strict Quality of Service (QoS) requirements of traffic safety applications will require vehicles to ubiquitously guarantee robust and reliable communication capabilities. As a result, the traffic safety applications requirements, together with the decentralized system operation, the high node's mobility and the potential channel congestion in highly dense traffic conditions, impose the need of advanced radio resource management techniques that efficiently employ the radio channel and ensure the system's scalability.

To achieve these objectives, several studies have proposed and demonstrated the potential benefits of adapting the vehicle's transmission parameters to the specific operating conditions. For example, the work in [1] proposed to adapt the transmission power level and other transmission parameters to reduce interference and increase the overall packet delivery ratio. The authors in [2] proposed a similar adaptation to increase the vehicular network connectivity. On the other hand, investigations such as those reported in [3] propose interesting approaches to reorganize the information to be transmitted based on its relevance and the vehicle's situation in order to improve the system performance and scalability. Despite all these efforts, it is important to stress that adaptive transmissions policies should not only work at optimizing the network capacity, throughput and/or connectivity, but also at guaranteeing the instantaneous traffic applications QoS requirements, which can be particularly challenging for traffic safety applications. This approach is followed for example in [4], where the authors propose a distributed power control algorithm that determines the optimum transmission power per vehicle to transmit periodic messages leaving enough available bandwidth for event-driven safety messages (i.e. messages resulting of the detection of hazards like a car's hard braking).

In this context, the authors proposed in [5] an adaptive transmission policy named OPRAM (OPportunistic-driven adaptive RAdio resource Management) that was designed to guarantee the traffic safety QoS requirements while reducing channel congestion and efficiently using the available channel resources. While [5] demonstrated OPRAM's traffic safety QoS performance benefits, [6] showed OPRAM's capability to reduce channel congestion and improve the system's scalability. Despite these promising results, this work shows that the performance of opportunistic adaptive transmission techniques like OPRAM can be significantly degraded under correlated radio channel propagation conditions. Although such correlation effects could be simplified for system level investigations, its impact on the instantaneous performance of adaptive communication policies cannot be neglected. As a result, this work proposes and evaluates various compensation policies that can efficiently overcome the negative effects caused by the radio channel correlation on the performance of adaptive vehicular communication techniques.

\section{RADIO CHANNEL MODELING}

The radio channel can have a significant impact on the performance of wireless vehicular communication systems, especially when considering traffic safety applications with low latency requirements [7]. In order to ensure the validity of any adaptive communications investigation, accurate radio propagation models for system level investigations taking into account the effects of pathloss, shadowing and multipath fading must be considered. While pathloss represents the local average received signal power relative to the transmit power as a function of the distance between transmitter and receiver, the

This work was supported in part by the Spanish Ministerio de Fomento under the project T39/2006, by the Spanish Ministerio de Industria, Turismo y Comercio under the CELTIC project CP5-013 / TSI-02400-2008-113 and by the Generalitat Valenciana under research grant BFPI06/126. 
shadowing models the effect of surrounding obstacles on the mean signal attenuation and the multipath fading effect results from the reception of multiple replicas of the transmitted signal at the receiver.

In this work, a detailed urban micro-cell propagation model developed in the WINNER project [8] has been considered to accurately model the radio propagation effects at the $5 \mathrm{GHz}$ band. Despite not considering V2V communication scenarios, the operating conditions of the WINNER urban micro-cell model are, to the authors' knowledge, those that currently best fit the $\mathrm{V} 2 \mathrm{~V}$ communications scenario given the unavailability of a complete $\mathrm{V} 2 \mathrm{~V}$ communication propagation model for system level investigations. The WINNER model also differentiates between LOS (Line-of-Sight) and NLOS (NonLine-of-Sight) propagation conditions. For LOS conditions, the WINNER pathloss is expressed as follows:

$$
P L_{L O S}(d[m])=\left\{\begin{array}{c}
22.7 \log _{10}(d[m])+41+20 \log _{10}(f[G H z] / 5) \\
\text { if } d<R_{b p} \\
40 \log _{10}(d[m])+41-17.3 \log _{10}\left(R_{b p}\right)+20 \log _{10}(f[G H z] / 5) \\
\text { if } d \geq R_{b p}
\end{array}\right.
$$

where

$$
R_{b p}=4 \frac{\left(h_{A}-1\right)\left(h_{B}-1\right)}{\lambda}
$$

$d$ is the distance between transmitter and receiver, $h_{A}$ and $h_{B}$ are their respective antenna heights and $f$ is the carrier frequency. For NLOS conditions, the pathloss can be expressed as:

$$
P L_{N L O S}\left(d_{A}[m], d_{B}[m]\right)=P L_{L O S}\left(d_{A}[m]\right)+20-12.5 n_{j}+10 n_{j} \log _{10}\left(d_{B}[m]\right)
$$

where

$$
n_{j}=\max \left(2.8-0.0024 d_{A}[m], 1.84\right)
$$

and $d_{A}$ and $d_{B}$ are the transmitter and receiver distances to the closest intersection. The shadowing effect is modeled with a log-normal random distribution with standard deviation equal to $3 \mathrm{~dB}$ and $4 \mathrm{~dB}$ for LOS and NLOS conditions respectively in urban micro-cell scenarios [8]. To account for the shadowing spatial correlation, the Gudmundson model considering an exponential autocorrelation function [9] is employed in this work. This model describes the correlation of the shadowing process at a distance $d$ as:

$$
R_{y y}(d)=\sigma_{s}^{2} \cdot \exp \left(-\frac{|d|}{d_{0}}\right)
$$

where $\sigma_{\mathrm{S}}$ is the shadowing standard deviation and $d_{0}$ equals $D / \ln (2)$, with $D$ being the distance at which the normalised correlation is 0.5 .

Finally, the multipath fading effect has been modeled as a Ricean distribution for LOS and as a Rayleigh one for NLOS conditions [8]. In addition to propagation loses, this work models the probabilistic nature resulting from radio transmission effects through the inclusion of the PER (Packet Error Rate) performance as a function of the Signal to Interference and Noise Ratio (SINR) [10].

\section{OPRAM ADAPTIVE TRANSMISSION POLICY}

To support traffic safety applications requirements while efficiently using the radio resources, the authors proposed OPRAM [5]. The OPRAM technique adapts the vehicles transmission parameters based on its position and proximity to a potential dangerous traffic area. In particular, for traffic safety applications, the OPRAM mechanism adapts the transmission power and packet rate only in a small region, named AR (Algorithm Region), before the critical distance $(C D)$. This critical distance is the minimum distance to a potential collision area at which a warning message needs to be received in order to provide the driver with sufficient time to stop and avoid the accident. The distance $C D$ depends on the vehicle's speed $(v)$, the driver's reaction time $(R T)$ and the vehicle's emergency deceleration $\left(a_{\max }\right)$ which is here based on a uniform deceleration model.

A target scenario for OPRAM's application is intersections (see Figure 1). By modifying the communications parameters in AR, OPRAM aims to guarantee the successful reception from a potentially colliding vehicle of at least one broadcast safety message before reaching $C D$. As illustrated in Figure 1, OPRAM transmits $N_{T}$ broadcast safety messages in AR with an increased transmission power level. The transmission power in $\mathrm{AR}$ is equal to that needed to ensure that all $N_{T}$ messages are correctly received with an equal average probability $p_{e}$. As shown in Figure 1, guaranteeing the same packet reception probability $p_{e}$ for the $N_{T}$ broadcast safety messages requires their transmission at different power levels. Outside AR, OPRAM maintains a constant $0.25 \mathrm{~W}$ transmission power level and a constant packet transmission rate of 10 packets/s. These communication conditions are sufficient to guarantee a vehicle's connectivity with the vehicles located along the same street in a $150 \mathrm{~m}$ range under Line of Sight (LOS) propagation conditions, as established by the WAVE guidelines for cooperative collision warning applications [11]. By employing low transmission powers outside AR, OPRAM reduces interferences levels and results in a more efficient use of the communications channel.

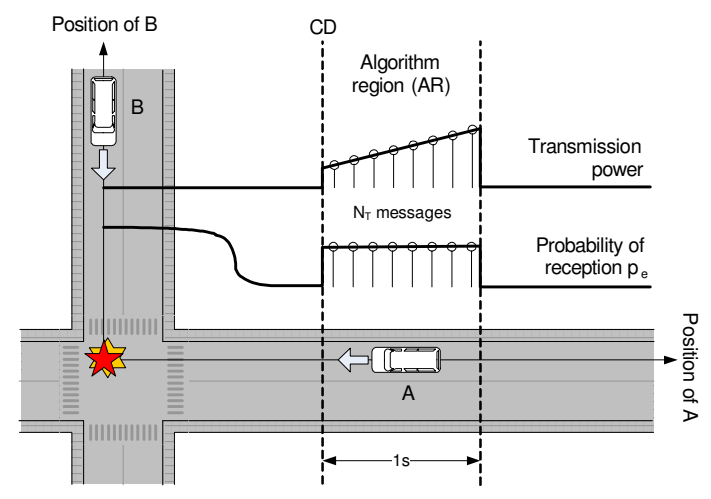

Figure 1. OPRAM configuration for traffic safety.

The probability that a single packet is successfully received in $\mathrm{AR}, p_{e}$, has been selected to ensure that at least one of the $N_{T}$ transmitted messages in AR is successfully received by the vehicle approaching the intersection, and that represents a potential collision risk, in $99 \%$ of the cases; this is equivalent to define a probability of not receiving a warning alert before 
$C D$ equal to $p_{n}=0.01$. The OPRAM proposal considers the probability $p_{e}$ to be constant in $\mathrm{AR}$, and independent for each one of the $N_{T}$ transmitted messages. In this case, the probability that no broadcast message is received from the potentially colliding vehicle before $C D$ is:

$$
p_{n}=\left(1-p_{e}\right)^{N_{T}}
$$

Having defined $p_{n}$ and $N_{T}, p_{e}$ can be obtained through equation (6). Once $p_{e}$ has been calculated, Figure 2 is used to obtain the required average received power level $\mathrm{Pr}$ to successfully receive each transmitted packet within AR with the probability $p_{e}$. Figure 2 has been obtained by separately evaluating a wide range of average received power levels, $P r$. For each of these average $P r$ values, a large set of instantaneously received power level samples is generated by adding to the average $\operatorname{Pr}$ value the shadowing and multipath fading contributions following their respective distributions. By computing SINR and using the corresponding PER curve, it can be decided whether each sample is correctly received or not. The probability of successfully receiving a packet $p_{e}$ given an average received power level $\mathrm{Pr}$ is then estimated as the ratio of correctly received samples to the total number of samples generated. It is interesting to note that the same relationship between the average probability $p_{e}$ and the average received power is obtained with and without considering the shadowing correlation effect in the random samples generation, given that Figure 2 represents average values. Once the mean $P r$ value necessary to guarantee the target $p_{e}$ has been determined, the needed transmission power can be obtained considering the distance between transmitter and receiver and the pathloss expressions previously provided.

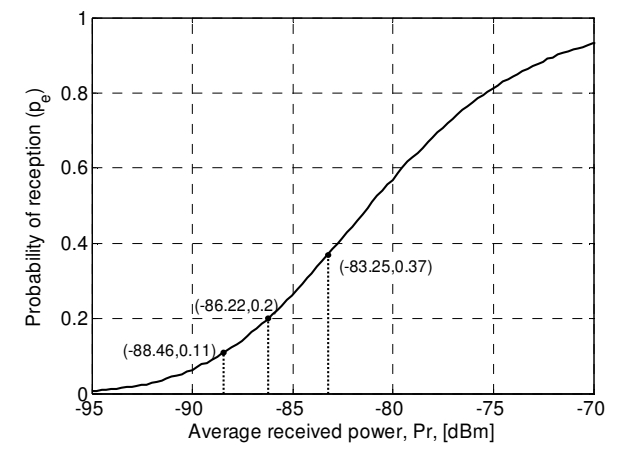

Figure 2. Average probability $p_{e}$ as a function of $P r$.

\section{OPRAM TRAFFIC SAFETY PERFORMANCE}

This work is based on the set of standards that are being developed to adapt the IEEE 802.11 operation to the vehicular environment: the IEEE 802.11 p or WAVE standard (Wireless Access in Vehicular Environments) [12], and the IEEE 1609 series of standards [13]. In the US, WAVE is based on seven ten-megahertz channels consisting of one control channel and six service channels in the $5.9 \mathrm{GHz}$ band. The service channels are used for public safety and private services, while the control channel is used as the reference channel to initially detect surrounding vehicles and establish all communication links. As a consequence, the control channel is used to periodically broadcast announcements of available application services, warning messages and safety status messages. In Europe, only recently $30 \mathrm{MHz}$ have been reserved for cooperative vehicular systems with the channel structure yet to be defined. WAVE makes use of the CSMA/CA medium access mechanism to grant the vehicles access to the channel. The ad-hoc mode is the only operational mode allowed in the WAVE control channel, which requires distributed radio channel management policies. It is important to note that the control channel's reference status to initiate any V2V and V2I communications or to detect the presence of a nearby vehicle could result in a high channel load in scenarios with a large number of nearby vehicles and broadcasted services. Such potential channel congestion, together with the strict traffic safety needs, requires the definition of advanced radio resource and channel management policies like OPRAM that efficiently use the WAVE control channel while guaranteeing the applications QoS and the system's scalability.

To evaluate the OPRAM traffic safety performance and efficiency, the Network Simulator ns2 has been employed emulating the critical intersection scenario illustrated in Figure 1. This scenario represents two vehicles moving towards an intersection with a risk of collision. To detect each other's presence, the vehicles periodically broadcast safety messages on the WAVE control channel at $6 \mathrm{Mbps}$, corresponding to the WAVE $1 / 2$ QPSK transmission mode. The vehicular speed has been set to $v=70 \mathrm{~km} / \mathrm{h}$ and the driver's reaction time to $R T=1.5 \mathrm{~s}$, which results in $C D=52.8 \mathrm{~m}$ and $C D+A R=72.2 \mathrm{~m}$. Using the OPRAM transmission power configuration based on the methodology previously described, Figure 3 depicts the resulting distribution of the number of broadcast safety alerts correctly received from the potentially colliding vehicle before $C D$ when the shadowing correlation is not modeled. The results shown in Figure 3 demonstrate that in this case OPRAM is capable to guarantee that $99 \%$ of the vehicles receive at least one broadcast safety message alerting of a potential road danger before reaching $C D$. Moreover, OPRAM satisfies the target traffic safety probability $p_{n}$ for varying values of $N_{T}$; following equation (6), an increasing value of $N_{T}$ results in a lower probability of reception $p_{e}$ and in significantly lower transmission power levels in AR.

Given that the OPRAM transmission mechanism considers the probability of reception of the $N_{T}$ packets transmitted in AR to be independent of each other when estimating the required transmission power levels, the radio channel correlation effect could significantly degrade OPRAM's traffic safety performance. While Figure 3 was obtained considering a correlation-free radio channel, Figure 4 presents the OPRAM traffic safety performance results when the shadowing spatial correlation effect is considered following the Gudmundson model. Radio channel correlation creates longer fading zones and burst errors and it increases the percentage of vehicles that are not capable to receive at least one broadcast safety alert before $C D$. As it can be observed in Figure 4, the higher probability of not receiving a safety alert before $C D$ due to the radio channel correlation effect is obtained for the higher $N_{T}$ values. In fact, the OPRAM configurations with higher $N_{T}$ values are those that required lower transmission power levels during AR. As a result, their performance exhibits a higher degradation when the shadowing correlation effect is modeled. 


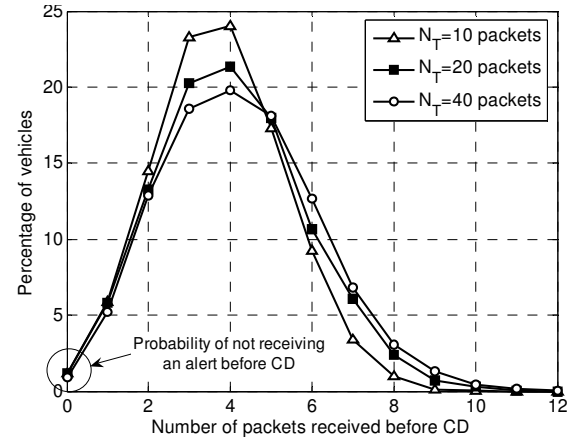

Figure 3. Percentage of vehicles that receive a given number of packets before $\mathrm{CD}$ without considering radio channel correlation.

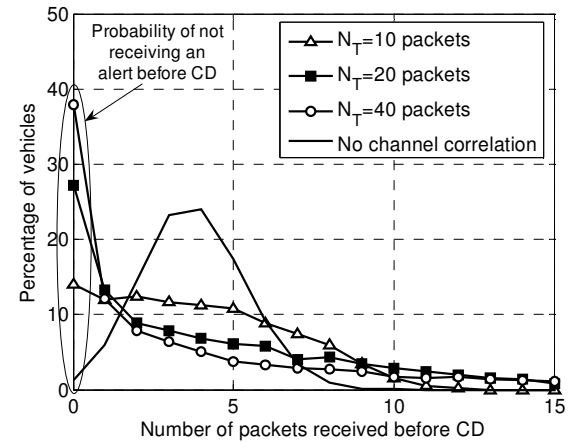

Figure 4. Percentage of vehicles that receive a given number of packets before $\mathrm{CD}$ considering radio channel correlation.

\section{OPRAM COMPENSATION POLICIES TO OVERCOME RADIO CHANNEL CORRELATION EFFECTS}

As previously explained, the OPRAM configuration methodology is based on the estimation of the transmission power level required to obtain the average probability of reception $p_{e}$ in each of the $N_{T}$ packets and guarantee the target traffic safety probability $p_{n}$. While this methodology was valid for correlation free propagation conditions, ensuring a given average value for the $p_{e}$ probability is not enough to satisfy the instantaneous traffic safety QoS requirements under correlated radio channels. In this context, this work proposes channel correlation compensation methodologies based on incrementing transmission power levels to ensure that only a given percentage of packets are received with probability lower than the target $p_{e}$ when the channel is correlated. Figure 5 shows the curve of the average probability $p_{e}$ as a function of the average received power, together with different probability percentile curves (each percentile curve corresponds to the probability that $\mathrm{x} \%$ of the transmitted packets are correctly received with a probability lower than $p_{e}$ ).

Having obtained $p_{e}$ with the original OPRAM methodology (equation (6)), using Figure 5 the reception power needed to guarantee that only the $\mathrm{x} \%$ of packets are received with probability lower than the target $p_{e}$ can be obtained. As an example, the white marks in Figure 5 illustrate the average reception power levels needed to guarantee that only $40 \%$ of packets are correctly received with a probability lower than the target $p_{e}$ values obtained for various $N_{T}$ values (i.e. that $60 \%$ of packets are correctly received with probability higher than $p_{e}$ ).
In order to ensure that the probability of correctly receiving packets below $p_{e}$ is as low as possible, higher received and transmitted power levels are required. Figure 6.a shows the OPRAM transmission powers obtained following equation (6) if OPRAM was configured so that only $\mathrm{x} \%$ of packets are received with probability lower than the target $p_{e}$ instead of just trying to guarantee the initial average target probability $p_{e}$ as in section IV. This approach is considered to combat the channel correlation effects on OPRAM's performance depicted in Figure 4.

Another possibility to combat channel correlation effects depicted in Figure 6.b for different percentiles is to transmit with fixed transmission power levels in AR instead of a constant $p_{e}$ probability. In this case, the transmission power in $\mathrm{AR}$ is maintained constant and equal to the transmission power level calculated for the farthest distance in AR using the OPRAM original methodology. Contrary to the initial OPRAM proposal, the transmission power is not decreased as the vehicles approach the intersection and their relative distance decrease. Maintaining the transmission power constant in AR will then increase the probability of correctly receiving broadcast safety messages as the two vehicles approach the intersection.

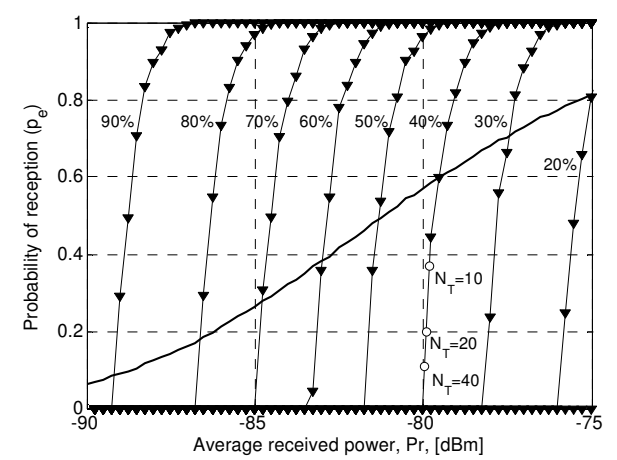

Figure 5. Average probability $p_{e}$ and corresponding percentiles as a function of $P r$.

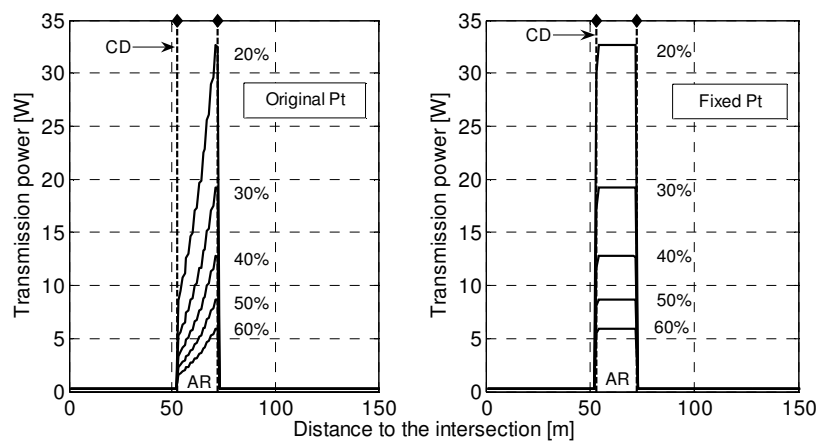

Figure 6. OPRAM channel correlation compensation techniques for different percentiles and $N_{T}=10$. (a) Original Pt. (b) Fixed Pt.

Using the OPRAM compensation techniques depicted in Figure 6, Figure 7 shows the percentage of vehicles that did not receive any broadcast safety message before $C D$ for varying percentiles and the $N_{T}$ values analyzed. First of all, it is important to note that the two proposed compensation 
techniques are capable to guarantee the target performance of $99 \%$ of vehicles receiving a broadcast safety alert before $C D$. From the results depicted in Figure 7, the maximum percentile (i.e. the minimum required transmission power configuration) that satisfies the traffic safety QoS requirements of $p_{n}=0.01$ can be obtained. As it can be observed, higher $N_{T}$ values require higher percentiles and lower transmission power levels. The fixed transmission power compensation policy shown in Figure 6.b results in higher average transmission power levels in AR compared with the original OPRAM compensation technique for the same percentile. As a result, Figure 7 shows that the fixed transmission power compensation technique can admit higher percentiles to satisfy the traffic safety QoS requirements. In this context, it is interesting to compare the transmission power levels required by the different OPRAM compensation techniques that are capable to guarantee the target probability $p_{n}=0.01$ under correlated channels.

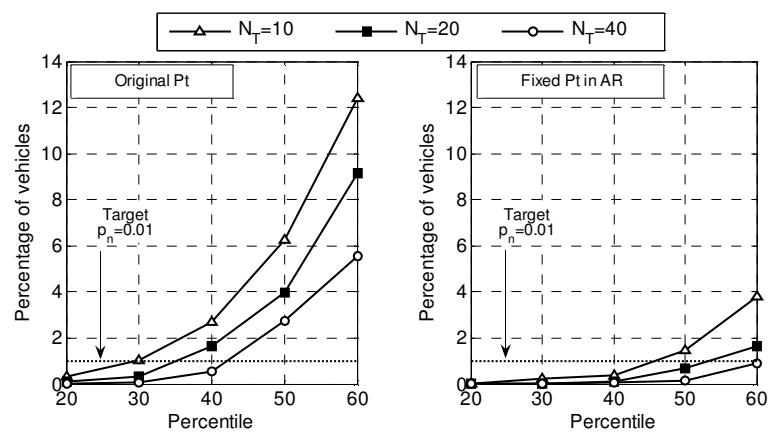

Figure 7. Percentage of vehicles that do not receive any message before CD considering radio channel correlation. (a) Original Pt. (b) Fixed Pt.

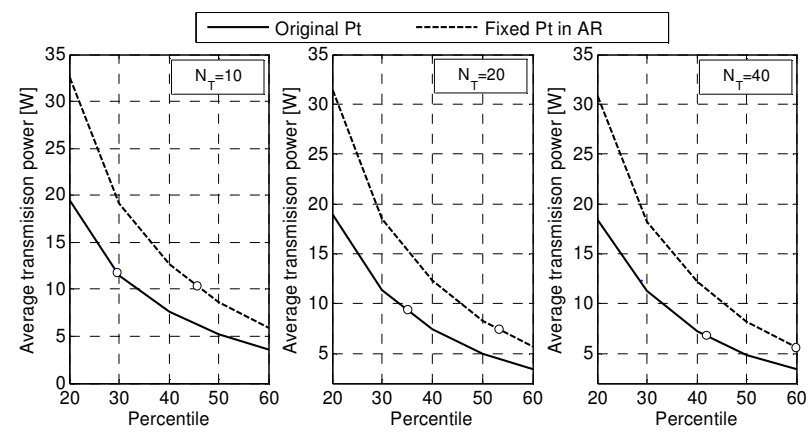

Figure 8. Average transmission power levels of the different OPRAM configurations analyzed.

Figure 8 shows the average transmission power levels of all the OPRAM compensation techniques analyzed. In this case, the white marks represent the maximum percentiles, extracted from Figure 7, that could be considered for the OPRAM compensation policies to be capable of guaranteeing the target traffic safety QoS level. Interestingly, the results illustrated in Figure 8 show that the OPRAM original configuration (constant probability of reception $p_{e}$ in $\mathrm{AR}$ ) requires higher average transmission power levels than the OPRAM fixed transmission power scheme (varying $p_{e}$ in $\mathrm{AR}$ ) to guarantee the same traffic safety QoS level when suffering from channel correlation. Given that the reduction of the transmission power diminishes the channel load and potential interferences, these results demonstrate the potential benefits of not considering a constant probability of reception in AR to overcome the radio channel correlation effects and satisfy the traffic safety QoS levels, while efficiently using the WAVE control channel. As depicted in Figure 8, increasing the number of packets $N_{T}$ transmitted in AR allows reducing the transmission power required to satisfy the traffic safety QoS requirements.

\section{CONCLUSIONS}

This paper has proposed and evaluated an opportunisticdriven adaptive transmission technique for wireless vehicular communication systems focused on traffic safety applications. The proposal has been evaluated under correlation-free and correlated radio channel conditions. While the original proposed methodology efficiently guarantees the traffic safety QoS requirements under correlation-free radio channels, the obtained results show that radio channel correlation can significantly degrade the proposal's performance. To this aim, two compensation policies designed to compensate the negative effects derived from the channel correlation have been proposed and evaluated showing their capability to guarantee the traffic safety QoS performance requirements.

\section{REFERENCES}

[1] M. Ruffini et al., "Power-rate adaptation in high-mobility distributed adhoc wireless networks", IEEE Vehicular Technology Conference VTCSpring, June 2005, Stockholm (Sweden), pp. 2299- 2303.

[2] G. Caizzone et al., "A power control algorithm with high channel availability for vehicular ad hoc networks", IEEE Int. Conference on Communications ICC, May 2005, Seoul (Korea), pp. 3171- 3176.

[3] T. Kosch et al., "The scalability problem of vehicular ad hoc networks and how to solve it", IEEE Wireless Communications Magazine, October 2006, pp. 22-28.

[4] M. Torrent-Moreno et al., "Distributed Fair Transmit Power Adjustment for Vehicular Ad Hoc Networks," IEEE Communications Society on Sensor and Ad Hoc Communications and Networks SECON, September 2006, Reston (USA), pp. 479-488 vol.2.

[5] J. Gozalvez and M. Sepulcre, "Opportunistic technique for efficient wireless vehicular communications", IEEE Vehicular Technology Magazine, December 2007, pp. 33-39 vol.2.

[6] J. Gozalvez and M. Sepulcre, "Channel efficiency of adaptive transmission techniques for wireless vehicular communications", 15th World Congress on Intelligent Transportation Systems, New York (USA), November 2008.

[7] M. Sepulcre and J. Gozalvez, "On the importance of radio channel modeling for the dimensioning of wireless vehicular communication systems", 7th International Conference on ITS Telecommunications ITST, June 2007, Sophia Antipolis (France), pp. 207-211.

[8] WINNER, "D1.1.1. WINNER II interim channel models", Public Deliverable. Online: https://www.ist-winner.org/

[9] M. Gudmundson, "Correlation model for shadow fading in mobile radio systems”, Electronic Letters, 27(23), November 1991, pp. 2145-2146.

[10] Y. Zang et al., "An error model for inter-vehicle communications in high-way scenarios at $5.9 \mathrm{GHz}$ ", Workshop on Performance evaluation of wireless ad hoc, sensor, and ubiquitous networks PE-WASUN, October 2005, Montreal (Canada), pp. 49-56.

[11] Vehicle Safety Communications Consortium, "Vehicle safety communications project task 3 - Final Report: Identify intelligent vehicle safety applications enabled by DSRC", DOT HS 809 859, March 2005.

[12] "IEEE P802.11p/D4.0: Part 11: Wireless LAN Medium Access Control and Physical Layer specifications. Amendment 8: Wireless Access in Vehicular Environments", IEEE Standards Association, March 2008.

[13] IEEE 1609, "Trial-Use Standard for Wireless Access in Vehicular Environments (WAVE)”, IEEE Standards Association, 2006. 